\title{
ESTUDIANTES EN RIESGO DE EXCLUSIÓN EDUCATIVA EN SECUNDARIA. PERCEPCIONES DEL PROFESORADO IMPLICADO EN PROGRAMAS EXTRAORDINARIOS DE PREVENCIÓN DEL FRACASO ESCOLAR
}

\author{
Students at risk of educational exclusion in secondary \\ education. Perceptions of teachers involved in special \\ programs about prevention of school failure
}

Maximiliano Ritacco ReAL* y Francisco Javier AmORes FERnÁNDEZ***

* Doctor en Ciencias de la Educación. Facultad de Ciencias Sociales y Humanas. Universidad de Zaragoza. Correo-e: ritacco@unizar.es

* Doctor en Ciencias de la Educación. Facultad de Ciencias de la Educación de la Universidad de Cádiz. Correo-e: **anranciscojavier.amores@uca.es

Recibido: 14-07-2015; Aceptado: 20-11-2015; Publicado: 30-05-2016

BIBLID [2386-3919 (2016) 34, 1; 137-160]

Ref. Bibl. M. RITACCO REAL y F. J. AMORES FERNÁNDEZ. Estudiantes en riesgo de exclusión educativa en secundaria. Percepciones del profesorado implicado en programas extraordinarios de prevención del fracaso escolar. Enseñanza \& Teaching, 34, 1-2016, 137-160.

RESUMEN: El fracaso escolar en la ESO es uno de los problemas más graves que arrastra el sistema educativo español en la actualidad. Los programas de cualificación profesional inicial (PCPI) se diseñaron para ofrecer una segunda oportunidad al alumnado fracasado (o en riesgo de exclusión educativa) en esta etapa educativa. Este estudio, para el que se ha utilizado un método mixto o híbrido de investigación, ha proporcionado una serie de resultados que ofrecen una visión más completa sobre el funcionamiento de este tipo de programas de «reenganche educativo» (ahora Formación Profesional Básica), en los centros de la provincia de Granada, mostrando puntos de vista tan interesantes como los del profesorado y alumnado implicados en 
MAXIMILIANO RITACCO REAL Y FRANCISCO JAVIER AMORES FERNÁNDEZ ESTUDIANTES EN RIESGO DE EXCLUSIÓN EDUCATIVA EN SECUNDARIA. PERCEPCIONES DEL PROFESORADO IMPLICADO EN PROGRAMAS EXTRAORDINARIOS DE PREVENCIÓN DEL FRACASO...

el estudio. Aquí se resume la percepción del profesorado acerca de estos alumnos en riesgo, que por razones diversas se han visto excluidos del sistema educativo ordinario y abocados al fracaso. Estudiantes que fracasan no sólo por dificultades de aprendizaje o por problemas personales relacionados con su entorno familiar, sino que también se estrellan contra un sistema educativo que no ha sido capaz de proporcionar las respuestas adecuadas a sus necesidades.

Palabras clave: Secundaria; alumnos en riesgo; exclusión educativa; fracaso escolar.

SUMMARY: School failure in Secondary Education is one of the most serious problems that carries the Spanish educational system today. Initial vocational qualification programs (PCPI) were designed to offer a second chance to failed students (or at risk of educational exclusion) in this educational stage. This study, for which has been used a mixed or hybrid method research, has provided a number of results that offer a more complete vision on the usefulness of these "Educational reclosing" programs (now Vocational Basic Training), in the centers of the province of Granada, showing viewpoints as interesting as the teachers and students involved in the study. Here the perception of teachers is summarized on these students at risk who, due to several reasons, have been excluded from the mainstream educational system, and doomed to failure. Students that fail not only for learning difficulties or personal problems related to their family, also crash into an educational system that has not been able to provide adequate answers to their needs. failure.

Key words: Secondary Education; students at risk; educational exclusion; school

\section{INTRODUCCIÓN}

Son muchas las medidas que se han venido aplicando para el conocimiento de los factores que inducen al fracaso escolar. Sin embargo, está pendiente un acercamiento más fino a las dimensiones generales y contextuales del riesgo escolar en diferentes contextos geográficos y centros educativos, a sus trayectorias personales y al diseño, desarrollo y resultados de las correspondientes medidas de respuesta.

En este sentido, y en referencia al estado de la cuestión, el concepto de alumnos en riesgo, que es objeto de un amplio estudio en la literatura especializada (Croninger y Valerie, 2001; Klasen, 1999; Vélaz de Medrano, 2005; Vidal, 2009; Zyngier, 2011), alude a aquellos estudiantes que encuentran a lo largo de su escolaridad dificultades acusadas para seguir con provecho el currículo y la enseñanza regular.

Tomando como referencia la UE, el Sistema Educativo español ofrece tasas de fracaso escolar en la educación obligatoria más altas que la media, aunque hay diferencias significativas entre las diferentes comunidades autónomas (Calero, 2007; IVEI, 2007; Navarrete, 2007; Teese, 2006). Igualmente, los índices de repetición, no 
idoneidad, promoción social, absentismo y abandono del sistema sin la titulación debida a la edad de dieciocho años nos sitúan, por desgracia, entre los primeros puestos de los mismos países de referencia, como muestra el Informe fOESSA (Renes Ayala, 2008). Esta situación se agrava entre la población inmigrante.

El problema es suficientemente relevante y uno de los principales objetivos políticos en el ámbito de la educación en Europa. En España, desde mediados de los noventa se han venido aplicando diversas medidas extraordinarias de atención a la población escolar de mayor riesgo de exclusión y de fracaso, sin embargo, el problema persiste.

Programas de Refuerzo, Orientación y Apoyo, Compensación Educativa, Programas de Diversificación Curricular, aulas taller, Programas de Cualificación Profesional Inicial (ahora Formación Profesional Básica), y otros con denominaciones diferentes según unas u otras CC. AA., han representado, de un lado, políticas y prácticas de tipificación y categorización de alumnos y, de otro, su derivación hacia determinadas medidas escolares, con sus correspondientes diseños organizativos y curriculares, procesos de enseñanza y aprendizaje profesionales y, en algunos de ellos, prácticas de iniciación profesional en contextos de trabajo diferentes. Sin embargo, si se pretende obtener una mayor efectividad, se requiere una descripción y comprensión más profunda y precisa de algunas de las facetas de las medidas extraordinarias de atención a los estudiantes con dificultades más severas, así como, dada la diversidad de las políticas autonómicas, una cierta mirada de conjunto que nos ayude a entender a nivel macro y micro este tipo de políticas, su desarrollo y resultados.

Un objetivo prioritario de la Comunidad Autónoma de Andalucía en esta década debe ser acercarse a los objetivos fijados por la Unión Europea. Después de los resultados manifestados en PISA 2012 (MECD, 2013) se trata de garantizar el derecho a la educación de todos los alumnos, entendido como la adquisición de aquel conjunto de competencias necesarias para su realización personal, ejercer la ciudadanía activa e incorporarse a la vida adulta de manera satisfactoria. En suma, una sociedad que se pretenda mínimamente cohesionada no puede permitirse -como actualmente- dejar a más de un 30\% de la población sin una cualificación básica o con abandono escolar temprano.

En cuanto a los antecedentes en este ámbito de estudio, las investigaciones realizadas en España, en unos casos, se han centrado en una mirada más bien escolar, mientras que en otros se ha investigado la conformación y las respuestas al riesgo en otros contextos formativos, concretamente referidos a la cualificación profesional. Conocer a nivel macro y micro la realidad y la respuesta al riesgo de exclusión educativa y social requiere analizar y comprender mejor cómo se construye y cómo se responde a ese fenómeno, valorar condiciones, procesos y resultados y difundir buenas prácticas para actuaciones sucesivas, ya que los datos disponibles revelan que existen diferencias dignas de atención en distintas Comunidades Autónomas (Escudero y Bolívar, 2008). 


\section{0}

MAXIMILIANO RITACCO REAL Y FRANCISCO JAVIER AMORES FERNÁNDEZ ESTUDIANTES EN RIESGO DE EXCLUSIÓN EDUCATIVA EN SECUNDARIA. PERCEPCIONES

DEL PROFESORADO IMPLICADO EN PROGRAMAS EXTRAORDINARIOS DE PREVENCIÓN DEL FRACASO...

Estudiar el complejo y controvertido fenómeno del fracaso escolar lleva consigo acercarse a la problemática realidad de la exclusión social y educativa. En efecto, en los últimos años se ha producido un desplazamiento terminológico en el uso de la noción de fracaso escolar hacia la noción de exclusión educativa (Castel, 2004; Luengo, 2005) a la hora de conceptualizar un conjunto de realidades heterogéneas que rodean el universo conceptual del fracaso: bajos rendimientos académicos, absentismo escolar, desenganche afectivo de la escuela, abandono prematuro de la educación obligatoria sin la graduación correspondiente. Además de estas dimensiones que atañen directamente a los resultados y trayectorias de los estudiantes, también acoge en su interior manifestaciones de carácter personal o social (comportamientos) que la escuela, los docentes, las familias y la sociedad valoran como inadecuados o insatisfactorios, así como las vivencias y reacciones negativas de los estudiantes, sus familias y sus entornos más cercanos (Escudero y Bolívar, 2008; Schonert-Reichl, 2000).

El fenómeno del fracaso escolar es dinámico y complejo, con una gran variedad de vertientes y una amplia bibliografía relacionada. Un indicativo de la importancia del problema es el creciente número de investigaciones relacionadas con el fracaso y el abandono escolar: Bernardi y Requena (2010), Bolívar y López (2009), Calero, Chois y Waisgrais (2010), Casquero y Navarro (2010), Escudero (2005), Escudero, González y Martínez (2009), Escudero y Martínez (2012), Feito (2015), Fernández Enguita (2009), Fernández Enguita, Mena y Riviére (2010), Roca Cobo (2010).

Asimismo, existen numerosos estudios relacionados con los Programas de Garantía Social, los Programas de Cualificación Profesional Inicial y otras medidas de respuesta a la diversidad, como, por ejemplo: Bernard y Molpeceres (2006), Calvo, Rodríguez y García (2012), Cutanda (2014), Cutanda y González (2015), González y Moreno (2013), González y Porto (2013), Marhuenda (2006), Merino, García y Casal (2006), Moliner, Sales, Ferrández, Moliner y Roig (2012), Zacarés y Llinares (2006).

\section{El PROGRAMA DE CUALIFICACIÓN PROFESIONAL INICIAL EN LA COMUNIDAD AuTÓNOMA DE ANDALUCÍA}

Los PCPI suponían un recurso educativo (hasta el curso escolar 2013-2014, posteriormente sustituido por la Formación Profesional Básica regulada por la Ley Orgánica para la Mejora de la Calidad) que daba continuidad a la posibilidad que abrió la Ley Orgánica de Ordenación General del Sistema Educativo (LOGSE, 1990) al crear los Programas de Garantía Social (Zacarés y Llinares, 2006), constituyéndose así en una nueva alternativa para el rescate de aquellos alumnos que estaban en riesgo de abandono prematuro del sistema educativo. El PCPI se instauraba en el sistema con el fin de superar las principales limitaciones de los antiguos PGS, teniendo como prioridad reducir las altas de fracaso escolar, dotar a los jóvenes 
de una cualificación profesional de nivel que les permita acceder al mundo del empleo y posibilitar al alumno el acceso a Formación Profesional de Grado Medio.

Estos programas estaban regulados en la Comunidad Autónoma de Andalucía por la Orden de 24 de junio de 2008 con la finalidad de dar una oportunidad educativa a los alumnos en riesgo de fracaso escolar y abandono temprano del sistema.

El PCPI posibilitaba que estos alumnos alcanzaran competencias profesionales propias de una cualificación de nivel uno de la estructura actual del Catálogo Nacional de Cualificaciones Profesionales creado por la Ley de las Cualificaciones y de la Formación Profesional, así como que tuvieran la posibilidad de una inserción sociolaboral satisfactoria y ampliaran sus competencias básicas para proseguir estudios en las diferentes enseñanzas (LOE, art. 30.2).

El tipo de alumnado que accedía a este programa eran jóvenes en riesgo de exclusión formativa, cultural y socioemocional, necesitados de unas medidas específicas para aprender. Solían proceder de situaciones de fracaso, abandono o sobreprotección mostrando una actitud de indefensión aprendida para enfrentarse a la formación, a la búsqueda de empleo y al mundo adulto en general. Por regla general procedían de modelos inadecuados de comportamiento familiar, social, laboral, etc. Por tanto, llegando con una actitud de rechazo hacia el ámbito educativo. En respuesta a esta necesidad, el PCPI se implementaba como una medida de atención a la diversidad destinada a evitar el abandono escolar previo a la finalización de la EsO.

Uno de los rasgos que caracterizaban al PCPI era su organización modular. Se estructuraba en dos cursos académicos, el primero de carácter obligatorio organizado en módulos específicos ligados a la formación profesional ofrecida por el perfil de cada PCPI (incluía horas prácticas en empresas del sector profesional) y módulos de formación general (proyecto emprendedor, participación y ciudadanía, y libre configuración). El segundo curso, de carácter voluntario, estaba organizado en torno a tres módulos: de comunicación, social y científico-tecnológico. La principal novedad frente a los Programas de Garantía Social era la posibilidad de obtención de la titulación del Graduado en Secundaria.

Estos programas estaban organizados por familias profesionales y dentro de cada familia, por perfiles. En la siguiente tabla se especifican los perfiles de cada una de las familias profesionales de los PCPI en la Comunidad Autónoma de Andalucía: 
MAXIMILIANO RITACCO REAL Y FRANCISCO JAVIER AMORES FERNÁNDEZ ESTUDIANTES EN RIESGO DE EXCLUSIÓN EDUCATIVA EN SECUNDARIA. PERCEPCIONES DEL PROFESORADO IMPLICADO EN PROGRAMAS EXTRAORDINARIOS DE PREVENCIÓN DEL FRACASO...

TABLA 1

Familias y perfiles profesionales de los PCPI en Andalucía

\begin{tabular}{|c|c|}
\hline FAMILIAS PROFESIONALES & PERFILES PROFESIONALES \\
\hline Administración y Gestión & Auxiliar de Gestión Administrativa. \\
\hline Agraria & $\begin{array}{l}\text { Auxiliar de floristería. } \\
\text { Auxiliar de agricultura y transformación } \\
\text { agroalimentaria. } \\
\text { Auxiliar en viveros, jardines y parques. }\end{array}$ \\
\hline Artes Gráficas & Operario de reprografía. \\
\hline Comercio y Marketing & Auxiliar de comercio y almacén. \\
\hline Edificación y Obra Civil & $\begin{array}{l}\text { Ayudante de albañilería. } \\
\text { Auxiliar en operaciones de acabado y pintura. }\end{array}$ \\
\hline Electricidad y Electrónica & $\begin{array}{l}\text { Auxiliar de Instalaciones Electrotécnicas y de } \\
\text { Comunicaciones. }\end{array}$ \\
\hline Fabricación Mecánica & $\begin{array}{l}\text { Operario de Soldadura y Construcciones Metálicas y } \\
\text { Tecnoplásticas. }\end{array}$ \\
\hline Hostelería y Turismo & $\begin{array}{l}\text { Auxiliar de alojamientos turísticos y catering. } \\
\text { Auxiliar de servicios en restauración. } \\
\text { Ayudante de Cocina. }\end{array}$ \\
\hline Imagen Personal & $\begin{array}{l}\text { Auxiliar de Peluquería. } \\
\text { Auxiliar en Técnicas estéticas. }\end{array}$ \\
\hline Industrias Alimentarias & Operario de Industria Alimentaria. \\
\hline Informática y Comunicaciones & Auxiliar Informático. \\
\hline Instalación y Mantenimiento & Ayudante de Fontanería y Calefacción-Climatización. \\
\hline Madera, Mueble y Corcho & $\begin{array}{l}\text { Auxiliar de carpintería. } \\
\text { Operario del corcho. }\end{array}$ \\
\hline Marítimo Pesquera & Auxiliar de acuicultura. \\
\hline Química & Auxiliar de laboratorio e industria química. \\
\hline Textil, Confección y Piel & $\begin{array}{l}\text { Auxiliar de reparación de calzado, marroquinería y } \\
\text { realización de artículos de guarnicionería. } \\
\text { Auxiliar de tapicería y entelados. } \\
\text { Operaciones de cortinaje y complementos de } \\
\text { decoración. } \\
\text { Operaciones de lavandería y arreglos de artículos } \\
\text { textiles. }\end{array}$ \\
\hline $\begin{array}{l}\text { Transporte y Mantenimiento de } \\
\text { vebiculos }\end{array}$ & Auxiliar de mantenimiento de vehículos. \\
\hline Vidrio y Cerámica & Auxiliar de manufactura cerámica. \\
\hline
\end{tabular}

Nota: Información obtenida en la web de la Junta de Andalucía.

En el curso escolar 2011-2012 se matricularon en PCPI un total de 15.854 alumnos en toda la Comunidad Autónoma de Andalucía, el 63,05\% en primer curso y el 
MAXIMILIANO RITACCO REAL Y FRANCISCO JAVIER AMORES FERNÁNDEZ

ESTUDIANTES EN RIESGO DE EXCLUSIÓN EDUCATIVA EN SECUNDARIA. PERCEPCIONES

DEL PROFESORADO IMPLICADO EN PROGRAMAS EXTRAORDINARIOS DE PREVENCIÓN DEL FRACASO...

$35,95 \%$ en segundo curso. El 69,5\% de los matriculados fueron hombres y el 30,5\% mujeres. La provincia con mayor número de alumnos en PCPI en este curso escolar fue Sevilla con un total de 3.150 alumnos, seguida de Málaga con 2.796 y Cádiz con 2.687 alumnos. La provincia con menor número de alumnos en el programa fue Huelva con 1.095 matrículas.

En la provincia de Granada, donde se desarrolló el estudio, tuvo un total de 1.729 alumnos matriculados en PCPI, de los cuales el 65,5\% en primer curso y el $34,5 \%$ en segundo. De estos alumnos, el 73\% en centros públicos y el 27\% restante en centros concertados. Los centros privados no contemplaban entre su oferta educativa Programas de Cualificación Profesional Inicial. La mayoría de los alumnos matriculados fueron hombres $(70,67 \%)$.

A partir de este marco de referencia emerge el estudio que aquí se presenta, con la finalidad de evaluar el PCPI (ahora FPB) en la provincia de Granada, teniendo los siguientes objetivos:

a) Analizar la realidad socioeducativa del PCPI de los centros educativos de la provincia de Granada.

b) Conocer la percepción del profesorado que imparte el PCPI en relación con la utilidad del programa en el contexto socioeducativo actual, y centrando la atención en aquellos aspectos que condicionan o pueden determinar el éxito o el fracaso del programa.

\section{MÉTODO}

De acuerdo con los objetivos que guiaron el proceso de la investigación, se consideró oportuno utilizar una metodología híbrida o mixta (Creswell, 2003; Tashakkori y Teddlie, 2010), combinando métodos cuantitativos y cualitativos en el mismo trabajo. El estudio sigue un diseño secuencial con un estatus dominante del método cuantitativo sobre el método cualitativo utilizado: QUAN $\rightarrow$ qual.

Participaron 50 centros docentes con PCPI entre su oferta educativa, lo que representa un 90,9\% del total en la provincia de Granada. De estos centros un $67,3 \%$ eran públicos y el resto, concertados. La población que dio paso a la selección de la muestra cuantitativa del estudio fue constituida por todos los profesores (345) y alumnos (1729) que formaron parte del PCPI en la provincia de Granada durante el curso académico 2011-12.

Se accedió a toda la población para su participación, y se obtuvieron un total de 213 respuestas en el caso de los profesores del programa (el 62,1\% de la población) y de 803 respuestas en el caso de los alumnos $(46,4 \%)$. 
MAXIMILIANO RITACCO REAL Y FRANCISCO JAVIER AMORES FERNÁNDEZ ESTUDIANTES EN RIESGO DE EXCLUSIÓN EDUCATIVA EN SECUNDARIA. PERCEPCIONES DEL PROFESORADO IMPLICADO EN PROGRAMAS EXTRAORDINARIOS DE PREVENCIÓN DEL FRACASO...

\subsection{Instrumentos}

Para la realización de este trabajo se emplearon dos modelos de cuestionarios (escala tipo Likert), que respondieron a un proceso de elaboración propia para un mayor ajuste a los objetivos perseguidos y al propio contexto de la investigación.

El cuestionario dirigido al profesorado quedó configurado por 57 ítems organizados en 7 dimensiones: a) consideración e importancia del programa como medida de prevención del fracaso escolar, b) caracterización del alumnado (motivaciones, procesos y causas del abandono escolar temprano), c) administración educativa, d) planificación, currículum y desarrollo del proceso de enseñanzaaprendizaje, e) formación específica del profesorado, funcionamiento general del programa y f) aspectos para la mejora.

El cuestionario dirigido al alumnado quedó configurado por 29 ítems organizados en 4 dimensiones: a) vivencia de la escolaridad desde el PCPI, b) organización modular del programa, c) motivaciones y causas del fracaso escolar y d) perspectivas de futuro.

Para la validación de contenido de los dos modelos de cuestionarios se contó con el juicio de un grupo de 13 expertos en la temática que, de manera general, realizaron comentarios y observaciones de los distintos ítems contemplados. En conjunto, los jueces emitieron una valoración positiva de todos los ítems, ofreciendo algunas recomendaciones de agrupación o desglose de ítems, así como la clarificación de algunos enunciados. Tras la validación de jueces y partiendo de las observaciones y aportaciones recibidas, se procedió a introducir, suprimir y modificar diferentes aspectos de forma y contenido de los cuestionarios, pero sin que la estructura general de los cuestionarios se viera alterada.

Para determinar su fiabilidad se realizó un estudio piloto. Se pasaron los cuestionarios en dos centros, uno de ellos concertado, en el que participaron 30 alumnos de 2 grupos de diferentes perfiles profesionales de primer curso y 7 profesores. De otro lado, se aplicaron los cuestionarios en un centro público en el que participaron 30 alumnos de primer y segundo curso y 8 profesores de PCPI. El profesorado cumplimentó el cuestionario realizando observaciones sobre forma y contenido para detectar posibles errores, añadiendo algunos ítems, eliminando otros, etc. Con respecto a los cuestionarios dirigidos a los alumnos, el profesorado responsable fue anotando las posibles dificultades a la hora de cumplimentarlos. Los cuestionarios se elaboraron a partir de su aplicación al alumnado y de las observaciones y comentarios del profesorado de PCPI (muestra piloto).

En relación con la parte cualitativa de la investigación, el objetivo de entrevistar a los profesores de PCPI fue conseguir un mayor entendimiento y acercamiento a las respuestas ofrecidas por los profesores y los alumnos en los cuestionarios aplicados. El guion de entrevista (semiestructurada) fue elaborado a partir del primer análisis exploratorio de los datos cuantitativos. En base a este análisis, y teniendo en cuenta la literatura sobre la temática y las dimensiones de estudio de los cuestionarios, se delimitaron las cuestiones que guiaron las entrevistas realizadas. Estas 
MAXIMILIANO RITACCO REAL Y FRANCISCO JAVIER AMORES FERNÁNDEZ

ESTUDIANTES EN RIESGO DE EXCLUSIÓN EDUCATIVA EN SECUNDARIA. PERCEPCIONES

DEL PROFESORADO IMPLICADO EN PROGRAMAS EXTRAORDINARIOS DE PREVENCIÓN DEL FRACASO...

cuestiones se organizaron en torno a 6 apartados relacionados con las distintas dimensiones del cuestionario dirigido a los profesores del programa:

1. Datos personales y profesionales: este apartado de preguntas se corresponde con las variables demográficas y profesionales incluidas en la primera parte del cuestionario (sexo, edad, titulación académica, curso y módulo que imparte, perfil profesional, etc.).

2. Alumnos de PCPI: en este apartado se incluyen preguntas sobre las características de los alumnos de estos programas, causas y motivos del fracaso escolar, expectativas, etc.

3. Administración y currículum: en este apartado se incluyen preguntas sobre edad de acceso al programa, la duración y estructura, la organización modular del PCPI, sobre el aprendizaje de los alumnos, los objetivos y contenidos propuestos, las estrategias metodológicas, la evaluación y otros aspectos del currículum.

4. Centro educativo: en este apartado se plantean cuestiones como la integración del programa en la vida del centro escolar, la imagen de los profesores y alumnos implicados, la relación con las familias y su implicación en el centro.

5. Profesores: este apartado está orientado a conocer la opinión del profesorado acerca de la formación específica del profesorado del programa.

6. Valoración general: este bloque de preguntas pretende conocer la opinión acerca de la mejora del PCPI y su visión general del programa.

Teniendo en cuenta las variables anteriores, en este estudio no se buscó una representatividad estadística, sino que la intención fue recoger cada una de las diversas voces implicadas en los diferentes contextos.

\subsection{Análisis de los datos}

El tratamiento de los datos recabados de los cuestionarios se efectuó a través de técnicas cuantitativas, mediante el programa spss Statistics 20, referidas a las exigencias del modelo estadístico y a los objetivos de la investigación, por ello han sido tratados a dos niveles, descriptivo e inferencial: a) a nivel descriptivo se han utilizado las medidas de tendencia central, como representativas del conjunto de la distribución y las de variabilidad con el fin de conocer cómo se agrupan los datos, b) a nivel inferencial, se han desarrollado pruebas de contraste de tipo no paramétrico (tablas de contingencias chi-cuadrado de Pearson), así como otro análisis multivariante de interdependencia más complejo como el análisis factorial.

Por otra parte, el análisis de contenido de la información recogida en las entrevistas fue utilizado en distintas fases con el objetivo de apoyar los resultados obtenidos de los cuestionarios aplicados. En la primera fase se analizaron 
MAXIMILIANO RITACCO REAL Y FRANCISCO JAVIER AMORES FERNÁNDEZ ESTUDIANTES EN RIESGO DE EXCLUSIÓN EDUCATIVA EN SECUNDARIA. PERCEPCIONES

DEL PROFESORADO IMPLICADO EN PROGRAMAS EXTRAORDINARIOS DE PREVENCIÓN DEL FRACASO...

las transcripciones de las entrevistas en profundidad. En esta etapa, se aplicó el "análisis de contenido" con el objetivo de establecer unidades de registro ${ }^{1}$. En la segunda fase las unidades de registro ya extraídas fueron clasificadas y reducidas en indicadores o unidades de análisis ${ }^{2}$, todo ello para establecer una cierta organización de los mensajes de las notas de campo y transcripciones. Como resultado, se logró identificar y clasificar una serie de precategorías en donde se expresaban la visión y opiniones de los profesionales de las cuales surgió un sistema de categorías emergentes ${ }^{3}$. Por último, dentro de cada categoría, se estableció un índice de frecuencia categorial ${ }^{4}$ (IFC).

Además, se utilizó el programa MAXQDA 11 para aplicar un análisis vertical sobre la visión de cada uno de los entrevistados y un análisis horizontal que permitió establecer relaciones entre las distintas categorías y subcategorías emergentes.

En la siguiente tabla es posible observar los momentos clave, dentro del proceso de estructuración de las categorías, donde se utilizó el análisis de contenido.

TABLA 2

Fases del proceso de estructuración sometidas al análisis de contenido

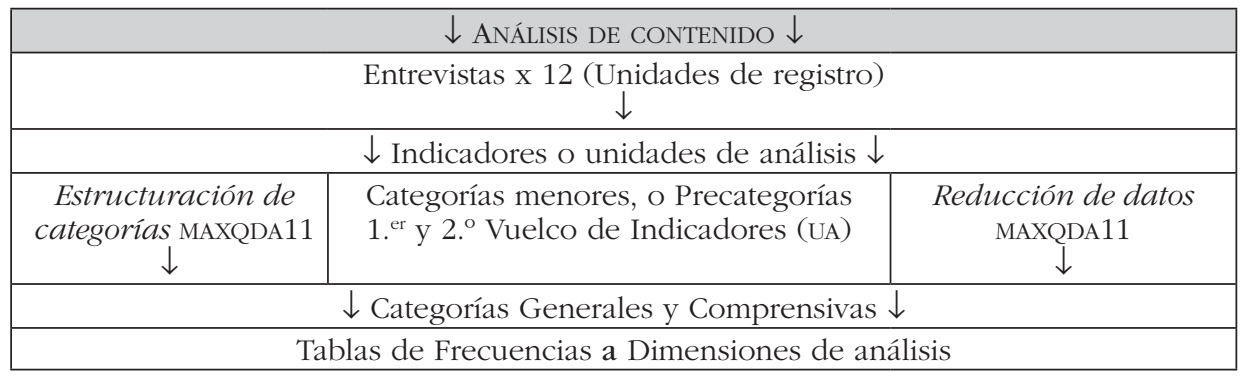

Nota: Elaboración propia.

1 Unidades de Registro: es la sección o segmento de contenido del texto que hace referencia a una categoría. Son unidades base con miras a la codificación, categorización y, si se precisa, al recuento frecuencial.

2 Los indicadores o unidades de análisis: constituyen los núcleos de significado propio que serán objeto de estudio para su clasificación en categorías.

3 Para Martínez (2006) la condición previa de todo proceso de categorización es «sumergirse» mentalmente en la realidad expresada buscando captar aspectos o realidades nuevas que enriquecen el significado. Para ello, el uso de esquemas de interpretación diseñando y rediseñando los conceptos resulta verdaderamente efectivo a la hora de "categorizar» (codificar mediante un término o expresión breve que sean claros e inequívocos) o clasificar las partes en relación con el todo.

4 El análisis de contenido da la posibilidad de establecer reglas de recuento (Índice de frecuencia categorial -IFC-) sobre los indicadores. En este caso se utilizó la frecuencia valorativa, que se refiere a la cantidad total de veces que un indicador se asocia con una serie de criterios determinados. 
ESTUDIANTES EN RIESGO DE EXCLUSIÓN EDUCATIVA EN SECUNDARIA. PERCEPCIONES

DEL PROFESORADO IMPLICADO EN PROGRAMAS EXTRAORDINARIOS DE PREVENCIÓN DEL FRACASO...

\subsection{Participantes. Identidad del colectivo docente y alumnado implicado}

En relación con el profesorado de PCPI de la provincia de Granada, según la variable "Sexo" en la muestra estudiada participaron 107 profesores $(50,2 \%)$ y 106 profesoras (49,8\%). En cuanto al curso académico participaron un 53,7\% de primer curso de PCPI, un $38,4 \%$ de segundo curso y un $7 \%$ que imparte clase en ambos cursos. El $21,1 \%$ del profesorado encuestado posee una experiencia profesional superior a 15 años. En relación con la experiencia docente en PCPI (o en Programas de Garantía Social), el 64,3\% posee menos de 5 años de experiencia, el 20,2\% entre 5 y 10 años y el 15,5\% restante más de 10 años de experiencia.

Respecto al alumnado de PCPI, según la variable sexo en la muestra estudiada participaron 587 chicos $(73,1 \%)$ y 216 chicas $(26,9 \%)$, de edades comprendidas entre los 15 y los 22 años. En relación con esta variable, el 73,8\% de los alumnos se encuentra en el intervalo de edad de 15 a 17 años y el $26,2 \%$ restante en el intervalo "de 18 a 22 años». En cuanto a la titularidad del centro participaron 554 alumnos de centros públicos (69\%) y 249 alumnos de centros concertados. Según la variable curso participaron 429 alumnos de primero de PCPI (61,5\%) y 309 alumnos de segundo $(38,5 \%)$.

Por otra parte, en relación con el estudio cualitativo, los sujetos entrevistados responden a unos perfiles previamente establecidos por los investigadores. La finalidad de la entrevista es obtener información complementaria del profesorado acerca de su propia visión sobre distintos aspectos del programa. Para alcanzar el nivel de saturación se entrevistó a un total de 12 profesores. Los criterios tenidos en cuenta a la hora de seleccionar los diferentes casos fueron: sexo, titularidad del centro, curso, módulo y materia que imparte, perfil profesional, años de experiencia docente y años de experiencia en este tipo de programas. Participaron 6 profesores y 6 profesoras con una amplia experiencia en este tipo de programas (entre 5 y 18 años), 5 profesores de centro público y 7 de centro concertado. Según la variable "curso", 7 profesores de primer curso y 5 de segundo.

\section{Resultados}

A continuación, se muestran los resultados de la dimensión de análisis centrada en la visión del profesorado de PCPI de la provincia de Granada sobre los alumnos del programa, las motivaciones, los procesos y las causas del fracaso y abandono escolar. En este sentido, la Tabla 3 recoge los resultados de los datos del cuestionario dirigido a los profesores sobre las cuestiones referentes al alumnado. Una vez analizada la visión del profesor acerca de las características de los alumnos de PCPI, se muestran las diferentes subcategorías surgidas del proceso de categorización de las entrevistas al profesorado con relación a diferentes aspectos del alumno: elección de itinerarios y expectativas; actitud, autoestima y comportamiento del alumno durante el desarrollo del programa; procesos del fracaso; motivaciones del fracaso; y por último las posibles causas del fracaso de estos alumnos. 
MAXIMILIANO RITACCO REAL Y FRANCISCO JAVIER AMORES FERNÁNDEZ ESTUDIANTES EN RIESGO DE EXCLUSIÓN EDUCATIVA EN SECUNDARIA. PERCEPCIONES DEL PROFESORADO IMPLICADO EN PROGRAMAS EXTRAORDINARIOS DE PREVENCIÓN DEL FRACASO...

TABLA 3

Opinión del profesorado sobre el alumnado de PCPI

\begin{tabular}{|c|c|c|c|c|c|c|}
\hline \multirow{2}{*}{ ÍTEMS } & \multicolumn{4}{|c|}{ VALORACIÓN } & \multirow{2}{*}{ M } & \multirow{2}{*}{$\mathrm{SD}$} \\
\hline & $\mathrm{N}(1)$ & A (2) & D (3) & $\mathrm{T}(4)$ & & \\
\hline $\begin{array}{l}\text { 1. Son como la mayoría, sólo que a lo largo } \\
\text { de su escolarización han encontrado } \\
\text { dificultades, sin resolverlas debidamente } \\
\text { a tiempo }\end{array}$ & $\begin{array}{c}14 \\
(6,6)\end{array}$ & $\begin{array}{c}75 \\
(35,2)\end{array}$ & $\begin{array}{c}76 \\
(35,7)\end{array}$ & $\begin{array}{c}48 \\
(22,9)\end{array}$ & 2,74 & 0,882 \\
\hline $\begin{array}{l}\text { 2. Tienen menos capacidades que las que } \\
\text { se requieren para cursar el currículo } \\
\text { ordinario de la ESO }\end{array}$ & $\begin{array}{c}72 \\
(33,8)\end{array}$ & $\begin{array}{c}90 \\
(42,3)\end{array}$ & $\begin{array}{c}39 \\
(18,3)\end{array}$ & $\begin{array}{c}12 \\
(5,6)\end{array}$ & 1,96 & 0,865 \\
\hline $\begin{array}{l}\text { 3. Tienen menos motivación e interés } \\
\text { pero no menos capacidades que sus } \\
\text { compañeros del aula ordinaria }\end{array}$ & $\begin{array}{l}15 \\
(7,0)\end{array}$ & $\begin{array}{c}62 \\
(29,1)\end{array}$ & $\begin{array}{c}79 \\
(37,1)\end{array}$ & $\begin{array}{c}57 \\
(26,8)\end{array}$ & 2,83 & 0,890 \\
\hline $\begin{array}{l}\text { 4. Tienen menos hábitos de estudio pero las } \\
\text { mismas capacidades que sus compañeros } \\
\text { del aula ordinaria }\end{array}$ & $\begin{array}{c}13 \\
(6,1)\end{array}$ & $\begin{array}{c}66 \\
(31,0)\end{array}$ & $\begin{array}{c}78 \\
(36,6)\end{array}$ & $\begin{array}{c}56 \\
(26,3)\end{array}$ & 2,83 & 0,890 \\
\hline $\begin{array}{l}\text { 5. Son estudiantes que no van a lograr los } \\
\text { aprendizajes y competencias básicas de } \\
\text { la Eso, haga lo que haga el profesorado }\end{array}$ & $\begin{array}{c}114 \\
(53,5)\end{array}$ & $\begin{array}{c}61 \\
(28,6)\end{array}$ & $\begin{array}{c}26 \\
(12,2)\end{array}$ & $\begin{array}{c}12 \\
(5,6)\end{array}$ & 1,70 & 0,892 \\
\hline $\begin{array}{l}\text { 6. Estos estudiantes obtendrían la titulación } \\
\text { de la Eso sin tener que recurrir a este tipo } \\
\text { de medidas, si en las aulas ordinarias se } \\
\text { seleccionara mejor los contenidos o } \\
\text { competencias claves }\end{array}$ & $\begin{array}{c}111 \\
(52,1)\end{array}$ & $\begin{array}{c}80 \\
(37,6)\end{array}$ & $\begin{array}{c}17 \\
(8,0)\end{array}$ & $\begin{array}{c}5 \\
(2,3)\end{array}$ & 1,60 & 0,736 \\
\hline $\begin{array}{l}\text { 7. Estos estudiantes podrían obtener } \\
\text { la titulación de Graduado sin tener } \\
\text { que recurrir a este tipo de medidas, si } \\
\text { en las aulas ordinarias se utilizaran } \\
\text { otras metodologías de enseñanza más } \\
\text { adecuadas a sus necesidades }\end{array}$ & $\begin{array}{c}75 \\
(35,2)\end{array}$ & $\begin{array}{c}89 \\
(41,8)\end{array}$ & $\begin{array}{c}34 \\
(16,0)\end{array}$ & $\begin{array}{c}15 \\
(7,0)\end{array}$ & 1,95 & 0,891 \\
\hline $\begin{array}{l}\text { 8. En el tiempo que he trabajado en este } \\
\text { Programa, he podido comprobar que } \\
\text { el alumnado vuelve a interesarse por el } \\
\text { trabajo escolar }\end{array}$ & $\begin{array}{c}6 \\
(2,8)\end{array}$ & $\begin{array}{c}77 \\
(36,2)\end{array}$ & $\begin{array}{c}93 \\
(43,7)\end{array}$ & $\begin{array}{c}37 \\
(17,1)\end{array}$ & 2,75 & 0,769 \\
\hline $\begin{array}{l}\text { 9. Lo que les hace falta a estos/as alumnos/ } \\
\text { as es un entorno social y familiar que les } \\
\text { estimule a estudiar }\end{array}$ & $\begin{array}{c}1 \\
(0,5)\end{array}$ & $\begin{array}{c}35 \\
(16,4)\end{array}$ & $\begin{array}{c}84 \\
(39,4)\end{array}$ & $\begin{array}{c}93 \\
(43,7)\end{array}$ & 3,26 & 0,744 \\
\hline $\begin{array}{l}\text { 10. Se piensa que el alumnado que termina } \\
\text { en el PCPI no puede ni quiere aplicarse al } \\
\text { estudio }\end{array}$ & $\begin{array}{c}65 \\
(30,5)\end{array}$ & $\begin{array}{c}71 \\
(33,3)\end{array}$ & $\begin{array}{c}57 \\
(26,8)\end{array}$ & $\begin{array}{c}20 \\
(9,4)\end{array}$ & 2,15 & 0,964 \\
\hline $\begin{array}{l}\text { 11. El alumnado de estos programas que } \\
\text { decide acceder al mundo laboral suele } \\
\text { encontrar trabajo relacionado con su } \\
\text { perfil profesional }\end{array}$ & $\begin{array}{c}35 \\
(16,4)\end{array}$ & $\begin{array}{c}128 \\
(60,1)\end{array}$ & $\begin{array}{c}41 \\
(19,2)\end{array}$ & $\begin{array}{c}9 \\
(4,2)\end{array}$ & 2,11 & 0,718 \\
\hline
\end{tabular}

Nota: Elaboración propia. 
Un alto porcentaje de los profesores opinan que los alumnos de PCPI son como la mayoría, aunque a lo largo de su escolarización han encontrado dificultades que no han podido resolver a tiempo provocando que estos alumnos tengan menos capacidades que las requeridas para cursar el currículo ordinario de la EsO. Esta realidad indica que en algún momento de la educación obligatoria el alumno se ha encontrado con obstáculos en el camino que no ha podido superar y que el centro no ha sido capaz de prever y solucionar. En este sentido, Escudero, González y otros (2013) afirman que «el riesgo o fracaso no pertenece en exclusiva a los estudiantes que no quieren o no pueden aprender. También concierne al sistema educativo, a los centros, profesorado y otros agentes escolares que también merecen ser interpelados y desafiados".

La visión que tiene el profesorado de los alumnos que cursan PCPI se ajusta a la realidad del problema objeto de estudio, son alumnos que han tenido alguna dificultad durante la educación obligatoria pero que por diferentes motivos no han sabido resolver a tiempo. En este proceso juega un papel muy importante el centro educativo, que no siempre está a la altura de las circunstancias y en lugar de solucionar el problema se convierte en parte del mismo. Además se observa que estos alumnos muestran menos motivación e interés, así como menos hábitos de estudio, pero no menos capacidades, que sus compañeros de la Eso. Estos alumnos que acaban en PCPI no pueden o no quieren aplicarse al estudio, por este motivo los profesores están divididos a la hora de determinar si estos estudiantes serán capaces o no de lograr los aprendizajes y competencias básicas de la ESO, independientemente de su labor como docentes y, de la misma manera, aproximadamente la mitad opina que si en las aulas ordinarias se seleccionaran mejor los contenidos o competencias claves, estos estudiantes obtendrían la titulación de la ESO sin tener que recurrir a este tipo de medidas. A pesar de todo se observa que el programa de PCPI consigue volver a atrapar la atención del alumno en los estudios, aunque para conseguirlo es fundamental que el estudiante se encuentre en un entorno social y familiar proclive.

\section{Discusión}

\subsection{El PCPI como medida de atención a la diversidad y prevención del fracaso escolar}

Fracaso y abandono temprano son dos fenómenos diferentes, pues uno se refiere a la educación obligatoria y el otro a la postobligatoria, pero el abandono temprano en España está condicionado en buena medida por el fracaso: aquellos alumnos españoles que al finalizar los estudios obligatorios no han obtenido el título de ESO, es decir, que fracasan, no pueden formalmente continuar estudios de bachillerato o de ciclos formativos de grado medio y, por tanto, parte del abandono temprano español se explica por esta razón (Roca Cobo, 2010). 
En el caso de los PCPI hay alumnos menores de 16 años que se encuentran ante su última oportunidad de conseguir el título de graduado en ESO, de manera que el PCPI sirve como herramienta para prevenir el fracaso escolar. Y, por otro lado, existen alumnos mayores de 16 años cursando PCPI para acceder a estudios superiores evitando el abandono temprano del sistema educativo, es decir, se cumple la otra finalidad de estos programas que es la del reenganche educativo de los alumnos en riesgo de exclusión educativa.

En este sentido, se considera un fracaso el abandono escolar temprano, definido por la Comisión Europea como el número de personas de 18 a 24 años con una educación secundaria básica como máximo y que no continúa estudiando, donde se incluye lo que estrictamente se considera fracaso (Roca Cobo, 2010).

La idea de partida es que el abandono escolar prematuro es el resultado de un proceso progresivo de desvinculación de la escuela por parte de aquellos que acaban abandonando antes de la obtención de un título postobligatorio (Mena Martínez, Fernández Enguita y Riviére Gómez, 2010).

Tal como describe González (2010 y 2013), el abandono está asociado a una serie de factores de riesgo que se pueden clasificar en dos categorías diferentes, por un lado, los factores de riesgo social, entre los que se incluyen aspectos como la raza/etnia, género, edad, lenguaje minoritario, estatus socioeconómico, estructura familiar, nivel educativo de los padres, lugar de residencia, etc. Estos factores sirven para caracterizar el riesgo de los alumnos de tener problemas relacionados con la escuela, de forma que cuanto mayor sea la acumulación de desventaja social asociada con estos factores mayor será el riesgo de fracaso. Y, por otro lado, existen los factores de riesgo académico asociados con problemas en la escuela, tales como bajas calificaciones y expectativas educativas, repetición de curso temprana, problemas de disciplina, etc., estos factores son indicativos de un futuro abandono escolar. En esta investigación se demuestra cómo en la mayoría de los casos estudiados el profesor observa una acumulación de estos factores de riesgo de abandono en los alumnos que cursan PCPI.

A continuación se detallan los factores identificados por los profesores entrevistados como causas probables de riesgo de fracaso escolar, y observamos una clara correlación con los factores descritos por la profesora González.

\subsection{Procesos del fracaso}

Los profesores entrevistados en el estudio hacen referencia a cuatro factores que intervienen en el proceso de desenganche progresivo del sistema educativo: absentismo escolar, problemas disciplinarios, repetición de curso y bajas calificaciones. 


\subsubsection{Absentismo escolar}

El «absentismo», referido a la etapa obligatoria, se asocia con aquellos alumnos que, estando escolarizados, tienen una asistencia irregular, en grados diversos. Como tal, el absentismo en dicha etapa es inaceptable, dado que todo alumno debe asistir a clase, por lo que la tolerancia debe ser cero (Bolívar y López, 2009). El primer paso para resolverlo es «visibilizar» el fenómeno, controlando y registrando regularmente la asistencia para identificar su magnitud, disponer de un registro acumulativo que permita ver su evolución e informar a las instancias locales oportunas (García Gracia, 2009). El absentismo escolar es uno de los principales factores de riesgo de fracaso escolar identificado por los profesores de los PCPI que han participado en el estudio.

[...] nosotros en este centro, en general lo que tenemos son: chicos de conducta disruptiva, de absentismo, de abandono, etc., o de familias muy desestructuradas, bastantes chicos con medidas judiciales también, de libertad vigilada, y luego, en menor medida, los chicos inmigrantes que nos mandan los centros de protección de menores.

El absentismo es un problema complejo que, como se observa en las entrevistas realizadas a los profesores, obedece a multitud de causas. Respecto a la solución del problema González sugiere que no hay una receta que pueda aplicarse con iguales efectos en todos los casos, la solución no radica exclusivamente en disponer de unos u otros reglamentos y procedimientos de actuación. Para paliar este problema se precisará de políticas integradas, tanto desde un punto de vista psicologista y asistencial como de tipo administrativo, coercitivo, siempre y cuando no se limiten a diseñar programas destinados a alumnos catalogados o etiquetados como «en riesgo", y siempre que los docentes cuenten con las competencias necesarias para ponerlos en práctica y estén comprometido con ellos.

\subsubsection{Problemas disciplinarios}

Los resultados de un estudio realizado por Mena Martínez, Fernández Enguita y Riviére Gómez en 2010, en el que los autores utilizaron la información académica, disciplinaria y de orientación correspondiente a 856 alumnos que habían abandonado los estudios antes de obtener un título postobligatorio en el curso 2007/08 en la provincia de Salamanca, muestran que el 28\% de los expedientes de alumnos que han abandonado prematuramente han tenido algún tipo de problema disciplinario. Los expedientes con medidas disciplinarias son especialmente frecuentes entre los que abandonan en primer ciclo de la ESO (llegan a ser el 60\%), siguen teniendo una importante presencia entre los que abandonan en el segundo ciclo (37\%) y son minoritarios en la etapa postobligatoria (8\%). La conclusión de este estudio es que quien tiene algún problema de disciplina, por leve que sea, tiende 


\section{2}

MAXIMILIANO RITACCO REAL Y FRANCISCO JAVIER AMORES FERNÁNDEZ ESTUDIANTES EN RIESGO DE EXCLUSIÓN EDUCATIVA EN SECUNDARIA. PERCEPCIONES

DEL PROFESORADO IMPLICADO EN PROGRAMAS EXTRAORDINARIOS DE PREVENCIÓN DEL FRACASO...

a tener resultados de fracaso en etapas obligatorias con mucha más frecuencia que el resto de los que no consiguen un título postobligatorio.

Uno de los profesores comenta que una de las causas de que el alumno termine en el PCPI es precisamente por problemas disciplinarios. En este sentido, el profesor entrevistado añade que los problemas de disciplina no deben ser la principal causa para destinar al alumno al PCPI.

Cada vez nos vamos encontrando con chicos con mayores problemas conductuales.

Los problemas disciplinarios son comunes entre los alumnos de PCPI, que a veces terminan en estos programas erróneamente pues cuentan con la suficiente capacidad para graduarse en ESO. Pero el sistema no es capaz de buscar una solución alternativa mejor que la de categorizarlo como "problemático" y enviarlo a un PCPI, sin embargo, el objetivo de estos programas no es el de corregir problemas de disciplina, sino prevenir y paliar el fracaso escolar y el abandono prematuro del sistema.

\subsubsection{Repetición de curso}

Uno de los factores individuales más relevantes que influyen en el rendimiento académico es la repetición de cursos (Calero, Choi y Waisgrais, 2010). El 42,3\% (MEC, 2008) de los alumnos españoles acumula retrasos antes de $4 .^{\circ}$ de ESO. Esta elevada cifra resulta de interés ya que la repetición de cursos está correlacionada positivamente con la probabilidad de fracaso escolar (Jimerson, Anderson y Whipple, 2002; Benito, 2007).

Son muchos los autores que coinciden en afirmar que la repetición de curso no parece ser una estrategia eficaz para reducir el riesgo de fracaso escolar (Calero, Choi y Waisgrais, 2010; Fuentes, 2009; Bolívar y López, 2009).

Los resultados indican que conseguir que los alumnos estén escolarizados en el curso que les corresponde resulta fundamental para que sus probabilidades de fracaso escolar se reduzcan. Este resultado respalda la recomendación de Fuentes (2009) acerca de la necesidad de reducir el número de repetidores en España.

La gran mayoría de los estudiantes de PCPI de la muestra ha repetido en alguna ocasión. Sobre todo, repiten sexto de educación primaria y algún curso en el primer ciclo de secundaria.

Son alumnos que han repetido varias veces porque no consiguen los objetivos de la etapa en ese momento, y se decide pasarlos al PCPI.

Teniendo en cuenta el análisis de las entrevistas, está clara la correlación entre repetición de curso y traspaso a programa de PCPI, todos los profesores entrevistados destacan como factor determinante para que un alumno no termine la ESO y acabe realizando un programa de PCPI la repetición de curso. Por tanto, esta no parece una medida que consiga el objetivo perseguido, es decir, reforzar 
los conocimientos del alumno para que pueda seguir cursando con normalidad la educación obligatoria.

\subsubsection{Situación académica}

La trayectoria académica de los alumnos que acaban matriculados en el PCPI está marcada por las bajas calificaciones, los suspensos, el abandono de asignaturas a las que no se presentan, repetición de curso y una desvinculación progresiva de la práctica educativa habitual. Todo esto ejerce una influencia en el alumno que termina en el programa con un nivel académico muy bajo.

[...] ellos no se sienten capaces de superar un examen, ni de superar una prueba, ni de superar un curso, porque toda su trayectoria ha sido suspender y suspender.

El nivel académico es muy bajo, la mayoría de estos alumnos adolecen de conceptos básicos que suponen un lastre de cara al rendimiento futuro. Sería conveniente fortalecer estos conocimientos antes de seguir avanzando en su formación.

\subsection{Motivaciones del fracaso: vivencia de la escolaridad}

Cuando hablan de su experiencia los alumnos que abandonan prematuramente el sistema educativo se detienen en su vivencia de la escolaridad, explican cómo lo que cotidianamente les acontecía dentro del centro afectó a su decisión. Todos concluyen que llegaron a la decisión de abandonar después de experiencias de fracaso, sea en el propio instituto o en primaria. El argumento es claro: si no he aprobado lo más fácil (primaria), para qué esforzarme en intentar aprobar lo que es más difícil (Mena, Fernández Enguita y Riviére, 2010). El profesorado entrevistado percibe que el alumnado que termina en el PCPI suele hablar de sus experiencias negativas en la etapa de secundaria, en concreto, experiencias traumáticas con algún profesor, las bajas calificaciones, la repetición de curso y otras vivencias que provocan una progresiva desvinculación o desenganche del entorno educativo generando en el alumno el rechazo total de la escuela.

Vienen muy machacados por la escuela estos niños y con una serie de aprendizajes de respuesta de supervivencia ante un sistema que ellos sienten que no los acoge.

La mayoría de los alumnos que abandonan la educación o la formación, una vez finalizadas las etapas de escolaridad obligatoria, encuentran insatisfactorio el entorno de aprendizaje, suelen presentar bajos resultados y una interacción negativa con los profesores (Roca Cobo, 2010). 
MAXIMILIANO RITACCO REAL Y FRANCISCO JAVIER AMORES FERNÁNDEZ ESTUDIANTES EN RIESGO DE EXCLUSIÓN EDUCATIVA EN SECUNDARIA. PERCEPCIONES DEL PROFESORADO IMPLICADO EN PROGRAMAS EXTRAORDINARIOS DE PREVENCIÓN DEL FRACASO...

\subsection{Causas del fracaso}

La paulatina resistencia del alumno a participar en el sistema educativo culmina generalmente en el fracaso escolar. Por una parte, el desinterés del alumno en participar activamente en su proyecto educativo y, por otra, la dificultad que éste puede entrañar para determinados perfiles de alumnos llevan a un desenlace de frustración y abandono. Otros aspectos de la vida del alumno influirán igualmente en su decisión de abandonar el sistema. La desestructuración familiar, en muchas ocasiones, unida al origen social son dos componentes básicos a tener en cuenta en el análisis de los problemas radicados en el origen del fracaso escolar. Pero no sólo éstos. El concepto de sí mismo vinculado en numerosas ocasiones a la etnia o la nacionalidad se unen al resto de las causas determinantes del fracaso que se han citado. Por último, se añade el perfil del alumno con necesidades específicas de apoyo educativo que hacen del sistema educativo un verdadero hándicap para éste.

Se suele hablar de tres perspectivas analíticas sobre el fracaso y el abandono escolares: la que se centra en los problemas de los alumnos, preocupada por la distribución de las capacidades, la motivación y el esfuerzo; la que se centra en factores sociales o culturales, como características de las familias, áreas desfavorecidas, etc., y la que se centra en el funcionamiento del sistema educativo, especialmente en torno al de los centros y al estilo didáctico de los profesores (Marchesi, 2003).

Es evidente que el fracaso escolar no se puede determinar específicamente en compartimentos estancos, sino que todas estas causas están interrelacionadas entre sí, las causas últimas del fracaso escolar están difuminadas.

\section{CONClusiones}

La visión del profesorado sobre su experiencia docente en estos programas ha sido muy reveladora. En términos generales, la valoración que realizan sobre la utilidad de los mismos como medida de prevención del fracaso escolar es positiva; sin embargo, un análisis más profundo evidencia los problemas asociados a su aplicación real en los correspondientes centros educativos. Este análisis ha contribuido a la identificación de las variables determinantes del éxito de los programas y los aspectos del mismo que deben ser objeto de mejora.

En términos generales, los profesores encuestados valoran los programas de forma positiva, pues los consideran una medida necesaria por su efectividad para reenganchar al alumno en el sistema educativo, opinan que los programas proporcionan a éstos una formación de base que les permite seguir estudiando y/o transitar al mundo laboral. Así mismo, también estiman su efectividad como medio para obtener el título de Graduado en EsO.

Con la aplicación de los programas se consiguen unos resultados satisfactorios de aprendizaje. Aprendizajes no sólo relacionados con los conocimientos académicos, sino con el comportamiento y la actitud de los alumnos, donde en muchos 
casos se encuentra el origen del problema del fracaso escolar. En este sentido, uno de los principales objetivos perseguidos por estos programas de atención a la diversidad es proporcionar al niño las habilidades necesarias para la vida en sociedad, contribuyendo a su maduración. Éste es uno de los principales éxitos conseguidos, junto con el reenganche del alumno al sistema educativo.

Los PCPI presentan, por su propio carácter preparatorio, un claro componente madurativo y de fomento del desarrollo positivo de los jóvenes participantes. Un estudio de Zacarés y Llinares (2006), con alumnos y profesores de PGS de la Comunidad Valenciana, concluía que los PCPI, lo pretendan o no explícitamente, se convierten en contextos de maduración personal que pueden tener una clara incidencia en las trayectorias vitales de los jóvenes que en ellos participen. Más allá de la finalidad cualificadora, los PCPI y otros recursos similares se constituirán en poderosas herramientas de "optimización evolutiva» (Viguer, 2004).

Los profesores destacan que los alumnos de PCPI parten de una mala experiencia educativa, bien porque no han podido adaptarse a las exigencias de la educación ordinaria, o bien porque han presentado problemas de disciplina, lo que ha degenerado en una falta de motivación y de interés por el estudio, así como en una baja autoestima. Por tanto, cuando el alumno llega por primera vez al programa, el profesor se encuentra en la necesidad de tener que abordar en primer lugar estos problemas antes de empezar a conseguir resultados de tipo académico.

En este sentido, en base a los resultados de las encuestas realizadas, se observa que un alto porcentaje de los profesores considera que los PCPI consiguen restablecer el interés del alumno por el estudio. La mayoría también opina que el éxito de los mismos radica en su capacidad para mejorar la autoestima y la imagen personal de los alumnos. Sin embargo, existe una división de opiniones en cuanto a si mejoran los hábitos de estudio.

En definitiva, hay mayor consenso acerca de la utilidad de los programas para solucionar problemas de actitud que sobre la utilidad de los mismos para solucionar dificultades de aprendizaje.

Las respuestas ofrecidas por el profesorado que ha participado en el estudio ayudan a delimitar el problema del fracaso escolar, pues aportan su visión sobre aspectos claves, como las motivaciones, el proceso y las causas del fracaso y abandono prematuro de la escuela. Esto contribuye a resolver una de las cuestiones primordiales planteadas en esta investigación: ¿por qué se produce el fracaso escolar?, la respuesta es bastante compleja y aunque no existe una única causa, la investigación ha servido para delimitar el problema, de cara a encontrar soluciones que permitan abordarlo en sus fases iniciales, reduciendo el número de alumnos que acaban en PCPI. De esta forma, el programa se convierte en la última oportunidad para aquellos alumnos en riesgo de exclusión educativa, cuando las medidas aplicadas durante la educación ordinaria no han funcionado.

Los profesores de PCPI perciben que sus estudiantes no son diferentes al resto, sus problemas se derivan de una falta de interés y de motivación, y no tanto de una menor capacitación, e inciden en la necesidad de inculcarles buenos hábitos 
MAXIMILIANO RITACCO REAL Y FRANCISCO JAVIER AMORES FERNÁNDEZ ESTUDIANTES EN RIESGO DE EXCLUSIÓN EDUCATIVA EN SECUNDARIA. PERCEPCIONES

DEL PROFESORADO IMPLICADO EN PROGRAMAS EXTRAORDINARIOS DE PREVENCIÓN DEL FRACASO...

de estudio. En este sentido consideran que los alumnos de PCPI están capacitados para obtener el graduado en ESO, aunque para ello necesitan de este tipo de programas, que consiguen captar de nuevo el interés por los estudios y motivar a unos alumnos que viven en un entorno social y familiar que no los estimula.

Sin embargo, sorprende comprobar la división de opiniones respecto a la afirmación "son como la mayoría, sólo que a lo largo de su escolarización han encontrado dificultades, sin resolverlas debidamente a tiempo". Relacionado con esta cuestión, y gracias a las entrevistas realizadas, se observa que el profesorado evita mencionar, como causa del fracaso escolar, las propias deficiencias del centro en particular, o del sistema educativo en general, y se centra más en las propias características del alumno. En una investigación similar, desarrollada sobre los Programas de Diversificación Curricular y de Garantía Social, Escudero y otros (2013) concluían:

Parece seguir presente la fuerte convicción de que es poco significativo lo que se puede hacer para que aprenda un sector del alumnado que llega a la ESO con malas trayectorias previas y que, además, pertenece a un medio familiar y social desfavorecido en lo económico, lo cultural y lo social. O se sale de ese círculo vicioso o por muchas cosas que se hagan y las buenas intenciones de ofrecer segundas oportunidades, el último tramo de la educación obligatoria seguirá siendo una zona de riesgos variados conducentes a resultados indeseables para bastantes chicos y chicas que lo cursan (p. 301).

La investigación que aquí se presenta deja claro un aspecto fundamental del problema estudiado y es que el fracaso escolar es el resultado de un proceso de desenganche progresivo del sistema educativo. La opinión expresada por el profesorado lleva a pensar que el PCPI, que debe ser la solución última, se termina convirtiendo en la única que se utiliza en realidad, desaprovechando las oportunidades de reenganche que se podrían aplicar en las fases tempranas de este proceso acumulativo de desafección escolar. Proceso que puede empezar con problemas de absentismo escolar, a veces incluso justificado por los propios padres de los alumnos, con problemas de disciplina, con una mala trayectoria académica, con bajas calificaciones, que acaba llevando al alumno a tener que repetir uno o varios cursos, lo que agrava el problema, pues está demostrado que aquellos que están escolarizados en el curso que les corresponde tienen mejores perspectivas de futuro, desde un punto de vista académico.

En este proceso de desafección escolar, los profesores señalan otros factores que inciden en el mismo, sobre los que se debería actuar, aunque a veces se necesite de la colaboración con otras instituciones públicas u organismos sociales, uno de los más importantes es el entorno social y familiar del alumno. Muchos de los jóvenes que acaban en PCPI proceden de familias desestructuradas o han vivido algún proceso traumático en su entorno familiar. Otro aspecto interesante, que se detecta en las entrevistas con los profesores, es la actitud de muchas de las familias de estos alumnos respecto a la educación, la escasa valoración que muchos padres 
conceden a una buena formación provoca que sus hijos abandonen prematuramente los estudios para insertarse de forma precaria en el mundo laboral. Además, la necesidad de los jóvenes de no destacar respecto del grupo de iguales es otro factor importante que puede abocar al alumno al fracaso escolar.

En todos los casos, los profesores que han participado en el estudio coinciden en la necesidad de enfocar la educación como un proceso flexible, motivador y adaptado a las necesidades de un alumnado que ya cuenta con carencias de aprendizaje por su propia trayectoria educativa y por su entorno social y familiar. En cualquier caso el PCPI debe dar respuesta a la diversidad alejándose de la rigidez del sistema educativo ordinario, y aunque debe ser consciente de la realidad de la situación educativa de los alumnos no puede ser simplemente un mero mecanismo para enseñar al alumno a saber-estar, a saber-hacer o a hacer ciudadanos adultos de pleno derecho, en cuyo caso lo único que lograría es reinsertar al alumno en el entorno social y laboral, pero con una escasa preparación académica, lo que normalmente lleva aparejado un futuro laboral precario. En este sentido, es fundamental conseguir el reenganche real al sistema educativo, ofreciendo al estudiante la posibilidad de acceder a una educación superior que sitúe al alumno de PCPI en igualdad de condiciones de cara a competir por un puesto de trabajo digno.

En definitiva, este tipo de programas deben estar concebidos como la última oportunidad que ofrece el sistema educativo para abordar el problema del fracaso, en ningún caso debe ser una vía de escape para excluir del sistema ordinario a aquellos alumnos con problemas de disciplina ni se debe utilizar para insertar en el sistema educativo a determinados alumnos con situaciones anómalas porque no ofrece otra alternativa, como el caso de los inmigrantes o de los jóvenes que tienen problemas con la justicia, pues el propósito perseguido es el de facilitar que el alumno pueda continuar estudiando.

\section{REFERENCIAS BIBLIOGRÁFICAS}

Benito, A. (2007). La LOE ante el fracaso, la repetición y el abandono escolar. Revista Iberoamericana de Educación, 43, 1-11.

Bernard i García, J. C. y Molpeceres Pastor, M. ${ }^{a}$ A. (2006). Discursos emergentes sobre la educación en los márgenes del sistema educativo. Revista de Educación, 341, 149-169.

Bernardi, F. y Requena, M. (2010). Inequality in educational transitions: the case of postcompulsory education in Spain. Revista de Educación, número extraordinario, 93-118.

Bolívar Botía, A. y López Calvo, L. (2009). Las grandes cifras del fracaso y los riesgos de exclusión educativa. Profesorado. Revista de Currículum y Formación de Profesorado, 13 (3), 51-77.

Calero, J. (Dir.) (2007). Desigualdades socioeconómicas en el sistema educativo español. Madrid: Ministerio de Educación y Ciencia.

Calero, J.; Chois, A. y Waisgrais, S. (2010). Determinantes del riesgo de fracaso escolar en España: una aproximación a través de un análisis logístico multinivel aplicado a PISA2006. Revista de Educación, n. ${ }^{\circ}$ extraordinario, 225-256. 
MAXIMILIANO RITACCO REAL Y FRANCISCO JAVIER AMORES FERNÁNDEZ ESTUDIANTES EN RIESGO DE EXCLUSIÓN EDUCATIVA EN SECUNDARIA. PERCEPCIONES DEL PROFESORADO IMPLICADO EN PROGRAMAS EXTRAORDINARIOS DE PREVENCIÓN DEL FRACASO...

Calvo Salvador, A.; Rodríguez Hoyos, C. y García Lastra, M. (2012). Lo mejor de todo es que nos escucháis. Investigar el aumento de la participación de los estudiantes en los Programas de Diversificación y de Cualificación Profesional Inicial. Revista de Educación, 359, 164-183.

Casquero Tomás, A. y Navarro Gómez, M. ${ }^{a}$ L. (2010). Determinantes del abandono escolar temprano en España: un análisis por género. Revista de Educación, número extraordinario, 191-223.

Castel, R. (2004). Encuadre de la exclusión. En S. Karsz (Coord.). La exclusión: bordeando sus fronteras. Barcelona: Gedisa

Creswell, J. (2003). Research design. Qualitative, quantitative and mixed methods approaches (2. ${ }^{\mathrm{a}}$ ed.). Sage: Thousand Oaks.

Croninger, R. y Valerie, L. (2001). Social Capital and Dropping Out of High School: Benefits to At-Risk Students of Teachers' Support and Guidance. Teachers College Record, 103 (4), 548-581.

Cutanda López, M. T. (2014). De los Programas de Cualificación Profesional Inicial (PCPI) a la Formación Profesional Básica: algunas consideraciones. Revista Educativa Hekademos, 16, 69-79.

Cutanda López, M. T. y González González, M. T. (2015). La colaboración entre docentes del programa de cualificación profesional inicial: el papel del departamento de orientación. Educatio Siglo XXI, 33 (2), 303-322.

Escudero Muñoz, J. M. (2005). Fracaso escolar, exclusión educativa: ¿Đe qué se excluye y cómo? Profesorado. Revista de Currículo y Formación del Profesorado, 1 (1), 1-24.

Escudero Muñoz, J. M. y Bolívar Botía, A. (Coords.) (2008). Respuestas organizativas y pedagógicas ante el riesgo de exclusión educativa. En X Congreso Interuniversitario de Organización de Instituciones Educativas (Actas). Madrid: Wolters Kluwer.

Escudero Muñoz, J. M.; González González, M. ${ }^{a}$ T. y Martínez Domínguez, B. (2009). El fracaso escolar como exclusión educativa: comprensión, políticas y prácticas. Revista Iberoamericana de Educación, 5, 41-64.

Escudero Muñoz, J. M. y Martínez Domínguez, B. (2012). Las políticas de lucha contra el fracaso escolar: ¿programas especiales o cambios profundos del sistema y la educación? Revista de Educación, número extraordinario, 174-193.

Feito Alonso, R. (2015). La ESO de adultos. Trayectorias de abandono escolar temprano entre estudiantes con experiencia laboral. Profesorado. Revista de Currículum y Formación del Profesorado, 19 (2), 351-371.

Fernández Enguita, M. (2009). Cuadernos de quejas. Revista de Libros, 148, 40-42.

Fernández Enguita, M.; Mena Martínez, L. y Riviére Gómez, J. (2010). Fracaso y abandono escolar en España. Barcelona: Fundación "La Caixa».

García Gracia, M. (2009). El absentismo escolar: algunas claves para el desarrollo de intervenciones integradas en el marco de una escuela inclusiva y del territorio. Monográficos Escuela, marzo, 4-6.

González González, M. T. (2010). El alumno ante la escuela y su propio aprendizaje: algunas líneas de investigación en torno al concepto de implicación. Revista Iberoamericana sobre Calidad, Eficacia y Cambio en Educación, 8 (4), 10-22.

González González, M. T. (2013). Absentismo escolar: posibles respuestas desde el centro educativo. REICE. Revista Iberoamericana sobre Calidad, Eficacia y Cambio en Educación, 12 (2), 5-27. 
MAXIMILIANO RITACCO REAL Y FRANCISCO JAVIER AMORES FERNÁNDEZ

ESTUDIANTES EN RIESGO DE EXCLUSIÓN EDUCATIVA EN SECUNDARIA. PERCEPCIONES

DEL PROFESORADO IMPLICADO EN PROGRAMAS EXTRAORDINARIOS DE PREVENCIÓN DEL FRACASO...

González González, M. T. (2015). La vulnerabilidad escolar y los programas de Cualificación Profesional Inicial. Apuntes para la formación profesional básica. Madrid: Wolters Kluwer.

González González, M. T. y Moreno Yus, M. A. (2013). El Programa de Cualificación Profesional Inicial: Entre la integración o la marginación en los contextos organizativos donde se desarrolla. Revista de Investigación en Educación, 11 (1), 118-133.

González González, M. T. y Porto Currás, M. (2013). Programas de Cualificación Profesional Inicial: valoraciones e implicación de los alumnos en la Comunidad Autónoma de Murcia. Revista de Educación, n. ${ }^{\circ}$ extraordinario, 210-235.

Instituto Vasco de Evaluación e Investigación Educativa (2007). Abandono Escolar en 3. ${ }^{\circ} y$ $4 .^{\circ}$ de la ESO. Bilbao: Departamento de Educación, Universidades e Investigación.

Jimerson, S. R.; Anderson, G. S. y Whipple, A. D. (2002). Winning the battle and losing the war: Examining the relation between grade retention and dropping out of high school. Psychology in the Schools, 39 (4), 441-457.

Klasen, S. (1999). Social Exclusion, Children and Education: Conceptual and Measurement Issues. OECD Reports.

Ley Orgánica 1/1990, de 3 de octubre, de Ordenación General del Sistema Educativo (LOGSE). BOE n. ${ }^{\circ} 238$ 04/10/1990.

Ley Orgánica 2/2006, de 3 de mayo, de Educación. BOE n. ${ }^{\circ}$ 108, 04/05/2006.

Ley Orgánica 8/2013, de 9 de diciembre, para la mejora de la calidad educativa. $B O E$ n. ${ }^{\circ} 295$, $19 / 12 / 2013$.

Luengo Navas, J. (Comp.) (2005). Paradigmas de gobernación y de exclusión social en la educación. Fundamentos para el análisis de la discriminación escolar contemporánea. Barcelona y México: Ediciones Pomares.

Marchesi, A. y Pérez, E. (2003). La comprensión del fracaso escolar en España. En A. Marchesi y C. Hernández Gil. El fracaso escolar. Madrid: Alianza.

Marhuenda Fluixà, F. (2006). Presentación. La formación para el empleo de jóvenes sin graduado: educación, capacitación y socialización para la integración social. Revista de Educación, 341, 15-34.

Martínez, M. (2006). La investigación cualitativa. Revista IIPSI, 9 (1), 123-146.

Merino, R.; García, M. y Casal, J. (2006). De los Programas de Garantía Social a los Programas de Cualificación Profesional Inicial. Sobre perfiles y dispositivos locales. Revista de Educación, 341, 81-98.

Ministerio de Educación, Cultura y Deporte (2013). PISA 2012. Programa para la evaluación internacional de los alumnos. Informe español. Volumen I: Resultados y contexto. Madrid: MECD.

Moliner García, O.; Sales Ciges, M. A.; Ferrández Berrueco, R.; Moliner Miravet, L. y Roig Marzá, R. (2012). Las medidas específicas de atención a la diversidad en la Educación Secundaria Obligatoria (ESO) desde las percepciones de los agentes implicados. Revista de Educación, 358, 197-217.

Navarrete Moreno, L. (2007). Jóvenes y fracaso escolar en España. Madrid: Instituto de la Juventud.

Renes Ayala, V. (2008). Pobreza y marginación, ¿̇ocho millones de pobres? Documentación Social, 149, 93-128.

Roca Cobo, E. (2010). El abandono temprano de la educación y la formación en España. Revista de Educación, número extraordinario, 31-62. 
MAXIMILIANO RITACCO REAL Y FRANCISCO JAVIER AMORES FERNÁNDEZ ESTUDIANTES EN RIESGO DE EXCLUSIÓN EDUCATIVA EN SECUNDARIA. PERCEPCIONES DEL PROFESORADO IMPLICADO EN PROGRAMAS EXTRAORDINARIOS DE PREVENCIÓN DEL FRACASO...

Schonert-Reichl, K. (2000). Children and Youth at Risk: some Conceptual Considerations. The Pan-Canadian Education Research Agenda Symposium: Children and Youth At Risk.

Tashakkori, A. y Teddlie, C. (Eds.) (2010). Handbook of Mixed Methods in Social \& Behavioral Research. (2. ${ }^{a}$ ed.). Sage: Thousand Oaks.

Teese, R.; Aasen, P.; Field, S. y Pont, B. (2006). Equity in education thematic review: Spain, country note. OCDE.

Vélaz de Medrano, C. y De Paz Higuera, A. B. (2010). Investigar sobre el derecho, el deseo y la obligación de aprender en la sociedad del conocimiento. Revista de Educación, número extraordinario, 17-30.

Vidal Fernández, F. (2009). Pan y rosas. Fundamentos de exclusión social y empoderamiento. Madrid: Fundación FoEssa.

Viguer, P. (2004). Optimización evolutiva. Madrid: Pirámide.

Zacarés González, J. J. y Llinares Insa, L. (2006). Experiencias positivas, identidad personal y significado del trabajo como elementos de optimización del desarrollo de los jóvenes. Lecciones aprendidas para los futuros Programas de Cualificación Profesional Inicial. Revista de Educación, 341, 123-147.

Zyngier, D. (2011). (Re) conceptualising risk: left numb and unengaged and lost in a noman's-land o what (seems to) work for at-risk students. International Journal of Inclusive Education, 15 (2), 211-231. 